\section{E-Mobilität und Energiespeicher im Blickfeld der Technikfolgen- abschätzung und Systemanalyse Erste Analysen und zukünftige Forschungsaufgaben}

\section{von Torsten Fleischer und Marcel Weil, ITAS}

\begin{abstract}
Elektrische Energiespeicher, besonders solche für den Einsatz in Elektrofahrzeugen, sind in den Fokus der forschungspolitischen und öffentlichen Diskussion gerückt. Dabei zeigt sich, dass viele Fragen ihrer konkreten technischen Ausgestaltung, der Kontexte, in denen sie eingebettet sind, sowie der Potenziale und Folgen ihrer verbreiteten Anwendung noch unzureichend beantwortet sind. Einige davon werden im Beitrag aufgegriffen und Vorschläge für zukünftige Aufgabenstellungen der Technikfolgenabschätzung und Systemanalyse unterbreitet.
\end{abstract}

\section{Hintergrund}

Erklärtes Ziel der Bundesregierung ist es, die durch den Verbrauch fossiler Energieträger entstehenden Umweltbelastungen, insbesondere die $\mathrm{CO}_{2}$-Emissionen, zu minimieren. Ansätze dafür bestehen unter anderem. in der verstärkten Produktion von elektrischer Energie durch alternative Energiequellen (z. B. Windkraft, Photovoltaik), aber auch in der Nutzung elektrischer Energie im Mobilitätssektor.

Hinsichtlich einer kontinuierlichen und verlässlichen Energieversorgung birgt die wachsende Einspeisung von regenerativen Energiequellen neue Herausforderungen. Diese liegen v. a. begründet in der stark fluktuierenden Energieeinspeisung solcher Quellen, in starker Abhängigkeit von Wind bzw. Sonnenstrahlung sowie deren begrenzter Vorhersagbarkeit. Deshalb wird zukünftig mit dem Zubau weiterer Windparks bzw. Photovoltaikanlagen auch der Bedarf an neuen Versorgungsnetzstrukturen, aber auch an leistungsfähigen stationären Energiespeichern (und damit mehr Flexibilität im Lastmanagement) deutlich zunehmen.

Ein weiterer in der Zukunft stark wachsender Bereich für die Anwendung von elektro- chemischen Speichern ist die Elektromobilität. Deren Förderung wird von der Bundesregierung in den letzten Jahren mit großem Aufwand vorangetrieben. Motive für diese Anstrengungen sind neben den oben genannten Umweltschutzzielen eine angestrebte Diversifizierung der (bisher erdölbasierten) Endenergieträgerstruktur im Straßenverkehr, die Reduktion von Verkehrslärm und Beiträge zu einer nachhaltigen Entwicklung, aber auch wirtschaftspolitische Interessen und industriepolitische Aspekte. Die Bundesregierung hat sich das Ziel gesetzt, bis zum Jahr 2020 mindestens eine Million und bis 2030 sechs Millionen Elektrofahrzeuge auf die deutschen Straßen zu bringen. Dazu sind umfangreiche Aktivitäten gestartet worden, unter anderem ein Nationaler Entwicklungsplan Elektromobilität (Bundesregierung 2009), ein nationales Innovationsprogramm Wasserstoff- und Brennstoffzellentechnologie (BMVBS 2006) sowie ein 500-Millionen-Euro-Förderprogramm im Rahmen des Konjunkturpaketes II (BMWi 2009).

Trotz dieser zunächst auf technische Aspekte fokussierten Aktivitäten sowie weiterer langjähriger Forschungs- und Entwicklungserfahrungen in Deutschland, die weit ins letzte Jahrhundert zurückreichen, steht die Elektromobilität vor einer Reihe von noch zu lösenden Problemen. Diese sind teilweise wissenschaftlichtechnischer Natur, viele von ihnen gehen aber über rein technische Aufgaben weit hinaus und bleiben dennoch zugleich eng mit ihnen verbunden. Eine rechtzeitige und kreative Auseinandersetzung mit ihnen kann helfen, Innovationsbarrieren eher zu erkennen und sozial robustere Lösungen zu identifizieren, die Elektrofahrzeugen zu mehr werden lassen als ein Nischenprodukt. Zur Beantwortung dieser Fragen können auch Systemanalyse und Technikfolgenabschätzung Beiträge leisten. Einige davon sollen im Folgenden vorgestellt werden.

\section{Konzepte für Elektrofahrzeuge}

Eine zentrale Fragestellung ist die nach dem „basic design“ zukünftiger Elektrofahrzeuge. Hier finden sich heute sehr unterschiedliche Vorstellungen $^{1}$, die unter anderem durch sozioökonomische Faktoren wie Nutzergewohn- 
heiten und -erwartungen, Mobilitätsverhalten und diesbezügliche zukünftige Veränderungen sowie Zahlungsbereitschaften, aber auch durch technische Faktoren wie Anforderungen an die Betriebs- und Systemsicherheit beeinflusst werden. Viele konzeptionelle Überlegungen gehen heute vom dominanten Entwurfsparadigma des fünfsitzigen, urlaubstauglichen Familienfahrzeugs mit mindestens $500 \mathrm{~km}$ Reichweite aus - mit entsprechenden Auswirkungen auf Fahrzeuggewicht, Speichergröße und damit auch Fahrzeugkosten insgesamt. Von Interesse wird darum sein, inwieweit andere, den technischen und ökonomischen Limitationen elektrisch angetriebener Fahrzeuge eher Rechnung tragende Fahrzeugdesigns neben die klassischen Konzepte treten und diese gegebenenfalls substituieren werden. So kann man durchaus fragen, ob angesichts der von den meisten Fahrzeugen heute durchschnittlichen täglich zurückgelegten Distanzen im zweistelligen Kilometerbereich eine Speicherauslegung auf mehrere hundert Kilometer Reichweite eine „vernünftige“ Option ist, oder ob sich für den vergleichsweise seltenen Fall langer Fahrstrecken akzeptable Add-on-Lösungen vorstellen lassen und welche diese sein könnten. Aus analytischer Sicht wäre zunächst zu klären, mit welchem Untersuchungsdesign Fragestellungen dieser Art valide erfasst werden könnten, bevor ihnen eine stärker entwicklungsleitende Funktion zukommen kann.

Damit einher geht die v. a. für systemanalytische Untersuchungen und Szenarioentwicklungen wichtige Frage nach dem erwarteten Diffusionsverlauf von Elektrofahrzeugen. Wer werden die ersten Nutzer (,early adopters") von Elektrofahrzeugen sein? Wer sind die Zielgruppen dieser Produkte? Flottenbetreiber? Kleinhaushalte im urbanen Bereich? Mehrkindfamilien auf dem Lande? Wie können daraus belastbare Annahmen für die Struktur der zukünftigen Flotte an Elektrofahrzeugen und seiner zeitlichen Entwicklung gewonnen werden? Da die Ergebnisse solcher Überlegungen von präformierender Wirkung für Folgenszenarien aller Art sind, kommt ihnen selbst wie auch ihrer systematischen Reflexion eine besondere Rolle in der wissenschaftlich-technischen und der durch Wissenschaft beratenen politischen Diskussion zu.
Antworten auf diese Fragen sind nicht nur von akademischem und forschungspolitischem Interesse, sondern haben bei einer Reihe von Themen unmittelbar praktische Relevanz. Eine betrifft die nach dem Layout der zukünftigen Ladeinfrastruktur von Elektrofahrzeugen. Die gegenwärtige „Philosophie“ scheint das Laden von Fahrzeugen an der ,Steckdose“ - in der eigenen Garage oder auf dem eigenen Stellplatz - in den Vordergrund zu stellen. Aus dieser Setzung entsteht eine Reihe von Konsequenzen. Einerseits ist diese Situation nur bei einer bestimmten Zahl von Haushalten gegeben. Andere, urbanere Strukturen mit „Laternenparkern“ oder regelmäßig wechselnden Stellplätzen werden hierdurch zunächst nicht bedient. Dies kann durchaus als Innovationsbarriere wirken, da dadurch großen Teilen von sozialen Gruppen der Zugang zur Elektromobilität aufgrund von Infrastrukturmängeln erschwert wird. Geht man zugleich, wie oft postuliert, davon aus, dass Elektrofahrzeuge v. a. im urbanen Bereich die stärksten Potenziale für eine Marktdiffusion haben, da hier Lärmarmut und lokale Emissionsfreiheit besonders zum Tragen kommen, gewinnt diese Problematik noch an Bedeutung.

Eine weitere Folge dieser Setzung betrifft die Analyse von Netzkapazitäten bzw. die Planung von eventuell notwendigen Netzausbauten. In vergleichsweise dünn besiedelten, von Ein- und Zweifamilienhäusern geprägten Wohngebieten - der typischen "Garagengegend“ stellen sich Fragen nach Netzengpässen und deren Ausbaumöglichkeiten aus technischer und ökonomischer Perspektive ganz anders als in hoch verdichteten urbanen Räumen. Auch sind flexibel nutzbare Lade- und Abrechnungstechniken bei wechselnden Nutzern ganz anders zu diskutieren als bei einem Haushalt zuordenbaren Ladestationen.

Ebenfalls mit der Frage nach dem technischen Fahrzeugdesign verbunden ist die Wahl der geeigneten Kombination von Antriebskonzept und Speichersystem. Grundsätzlich müssen für mobile Anwendungen Energiespeichermedien entwickelt werden mit hohen Energie- und Leistungsdichten, einer langen Lebensdauer (kalendarische Lebenszeit, Zyklen-Lebenszeit; s. Böse 2009) sowie einer hohen thermischen 
Stabilität. Es stehen schon heute zahlreiche Materialsysteme (einschließlich ihrer Varianten) für Speicher zur Verfügung, die diese Kriterien in unterschiedlicher Güte erfüllen. Einige Materialsysteme werden im folgenden Kapitel benannt, eine ausführlichere vergleichende Darstellung findet sich in Oertel (2008). Zugleich sind bei den Automobilen mit Mikrohybrid, Mildhybrid, Vollhybrid, Plug-in-Hybrid und reinen Elektroautos unterschiedliche Antriebstypen in der Diskussion, die jeweils eine sehr unterschiedliche Performance von dem elektrischen Energiespeicher verlangen. Darüber hinaus hängt der Erfolg der entwickelten Energiespeicher nicht zuletzt von ihren Kosten sowie weiteren Systemanforderungen, etwa im Hinblick auf Betriebssicherheit und Schadensverhalten, ab. Die Entscheidung für ein bestimmtes Speichersystem ist mithin ein komplexes Auswahlproblem, dass in einem dynamischen technischen, ökonomischen und sozialen Umfeld zu lösen ist. Einen ersten Einblick in diesen Prozess soll das nachstehende Kapitel bieten.

\section{Technologischer Vergleich von elektrochemischen Speichersystemen}

Elektrochemische Speichersystem eröffnen die Möglichkeit, dass die bisher voneinander getrennten elektrische Energienetzwerke und Transportnetzwerke miteinander interagieren, wenn nicht sogar zukünftig teilweise miteinander verschmelzen. Beispielsweise können Elektrofahrzeuge durch die Erzeugung regenerativer (aber auch konventioneller) Energie angetrieben werden, oder für das Lastmanagement überschüssige regenerativ (oder konventionell) erzeugte Energie zwischenspeichern (soweit eine Netzanbindung besteht). Ferner können auch stationäre elektrisch Energiespeicher für die Schnellladung der elektrischen Energiespeicher in Elektrofahrzeuge herangezogen werden.

In den zusammenwachsenden Energie- und Transportnetzwerke gibt es zahlreiche unterschiedliche stationäre und mobile Anwendungen für Energiespeicher. Von großem Interesse ist dabei die Frage, welches der am Markt befindlichen elektrochemischen Speichern den Kundenerwartungen in einzelnen Anwendungsgebieten am ehesten gerecht werden kann, bringen diese Systeme doch jeweils deutlich unterschiedliche Performance hinsichtlich der Indikatoren Leistungs- und Energiedichte, Kosten, Sicherheit, Temperaturverhalten sowie Lebensdauer mit sich. Hinsichtlich dieser sechs Kriterien wurde in einer am ITAS durchgeführten Sondierungsstudie der Versuch unternommen folgende elf unterschiedliche elektrochemische Energiespeicher vergleichend qualitativ zu bewerten:

- Blei-Akkumulator

- Nickel-Cadmium Akkumulator

- Nickel-Metallhydrid Akkumulator

- Natrium-Nickelchlorid Akkumulator

- Natrium-Schwefel Akkumulator

- Vanadium-Redox-Flow Akkumulator

- Lithium-Ionen Akkumulator

- Lithium-Eisen-Phosphat Akkumulator

- Lithium-Mangan-Oxid Akkumulator

- Lithium-Polymer

- Doppelschichtkondensatoren (Supercap)

In Einzelanalysen wurden alle elf Energiespeicher auf Systemebene hinsichtlich der sechs Einzelkriterien qualitativ bewertet. Dabei erhält der beste Wert eines Einzelkriteriums (z. B. Energiedichte) die Punktzahl 6, der schlechteste Wert die Punktzahl 0,5 zugewiesen. Den Wertebereichen dazwischen werden in 0,5 Schritten die Punktzahlen (in der Regel) linear zugeordnet (vgl. Frankenberg 2010).

Soweit keine spezifische Anwendung im Focus der Betrachtungen ist, können für einen ersten Analysenschritt die erreichten Punktzahlen bei den einzelnen Kriterien ohne Gewichtung (d. h. Gleichgewichtung) addiert werden (vgl. Abb. 1).

Soweit ein spezifischer Anwendungsbereich betrachtet ist, wurden die Punktzahlen der Einzelkriterien mit einem Gewichtungsprofil (vgl. Abb. 2) multipliziert und die Einzelergebnisse zu einer Gesamtpunktzahl addiert (vgl. Abb. 3). Hierfür wurden acht stationäre und mobile Anwendungen exemplarische Gewichtungsprofile (vgl. Abb. 2) erstellt. Diese wurden mit Entwicklern von elektrochemischen Speichern innerhalb und außerhalb des KIT diskutiert und entsprechend angepasst. Die entwikkelten Gewichtungsprofile sind als vorläufiges 
Zwischenergebnis $\mathrm{zu}$ betrachten und müssen für weitere Vertiefungsarbeiten im Bezug auf die möglichen Anwendungsgebiete in einem breiteren Akteurskontext diskutiert werden.

In Abbildung 1 ist das Ergebnis des Vergleichs bei Gleichgewichtung dargestellt und zeigt deutlich die Stärken und Schwächen der einzelnen Batteriesysteme. Beispielsweise nimmt der Doppelschichtkondensator (auch Supercap genannt) trotz seiner deutlichen Nachteile bei den Systemkosten und der Energiedichte insgesamt eine Spitzenposition ein.

Neben den Supercaps gehören die NatriumSchwefel-, Vanadium-Redox-Flow-, NatriumNickelchlorid-, Lithium-Eisen-Phosphat- und insbesondere Lithium-Mangan-Akkumulatoren zu einer sehr aussichtsreichen Spitzengruppe.

Zweifellos ist das Ergebnis eines spezifischen Vergleichs entscheidend davon abhängig, welche Bedeutung z. B. die Akteure in Entscheidungsprozessen den einzelnen Kriterien für bestimmte Anwendungsgebieten einräumen. Über eine unterschiedliche Gewichtung der Kriterien kann man einer fallspezifischen Bedeutung Ausdruck verleihen.
Im Bereich der Elektromobilität werden jeweils unterschiedliche Anforderungen an elektrochemische Speicher in Hybridfahrzeugen, Plug-In-Hybridfahrzeugen und reinen Elektrofahrzeugen gestellt. Beispielsweise ist die Energiedichte der elektrochemischen Speicher bei reinen Elektrofahrzeugen sehr bedeutsam. Im Gegensatz dazu ist diese bei Hybridfahrzeugen weniger wichtig, dafür ist die Leistungsdichte von größerer Bedeutung.

Als Konsequenz dieser Überlegungen zeigen die Untersuchungen, dass für den Bereich der Hybridfahrzeuge mit Abstand die Supercaps, Lithium-Eisen-Phosphat- und insbesondere Lithium-Mangan-Akkumulatoren besonders aussichtsreich erscheinen. Dies mag überraschen, insbesondere da die für Hybridfahrzeuge aktuell am meisten eingesetzten Nickel-Metallhydrid Akkumulatoren (beispielsweise im Toyota Prius) nur den drittletzten Platz in dieser Untersuchung belegten.

Für reine Elektrofahrzeuge gibt es kein so klares Ergebnis (Abb. 3). Eine breitere Gruppe an aussichtreichen alternativen Speichertechnologien umfasst Natrium-Schwefel, Natrium-Nic-

Abb. 1: Vergleich der untersuchten elektrochemischen Energiespeicher im Fall einer Gleichgewichtung der Kriterien*

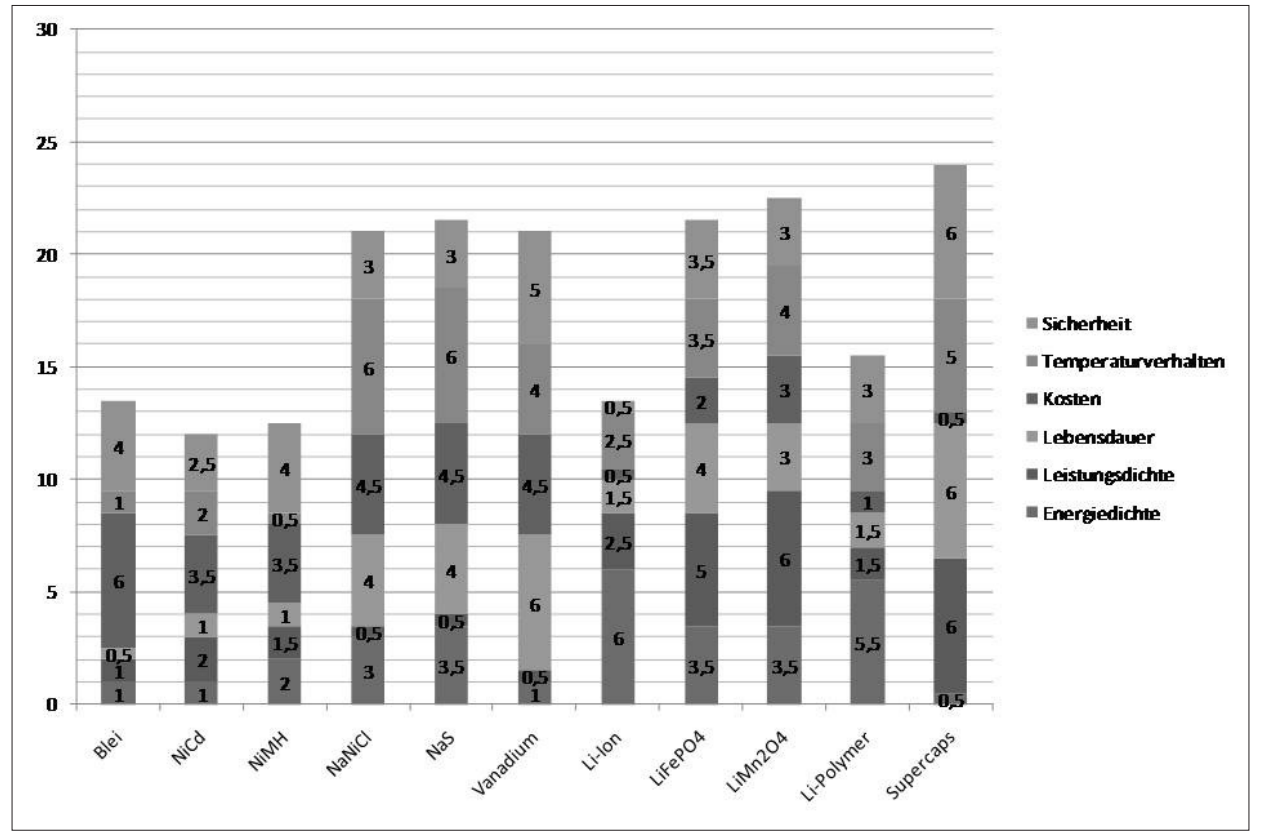

*= Die Y-Achse ist eine dimensionslose Punkteskala, vorteilhafte Energiespeicher weisen hohe Punkzahlen auf.

Quelle: Frankenberg 2010 
Abb. 2: Gewichtungsprofil der Kriterien für den Anwendungsbereich Elektrofahrzeuge

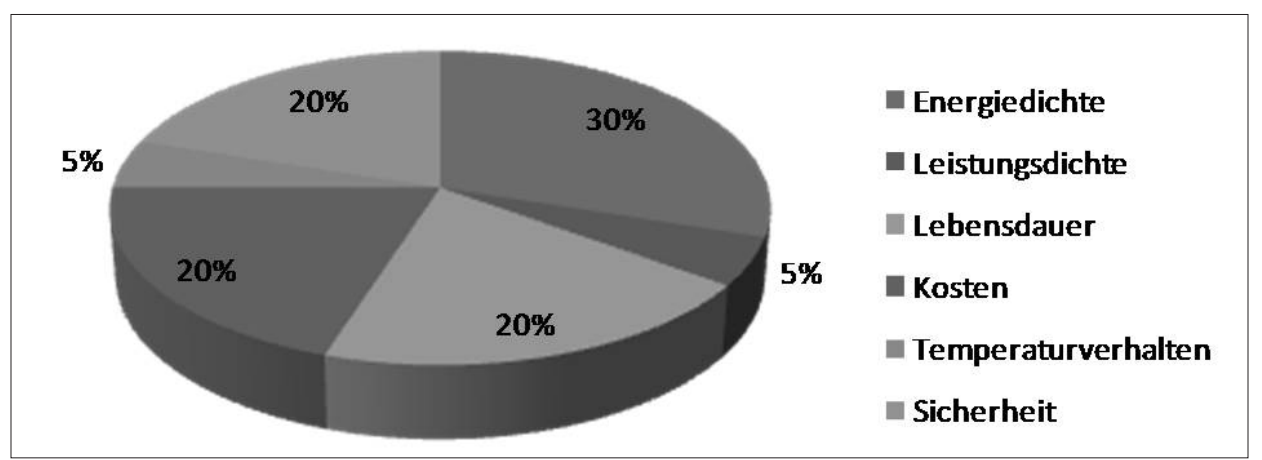

Quelle: Frankenberg 2010

kelchlorid-, Lithium-Ion-, Lithium-Polymer-, Lithium-Eisen-Phosphat- und nicht zuletzt Lithium-Mangan-Akkumulatoren.

Es zeigt sich also, dass im Bereich der mobilen Anwendung von elektrochemischen Speichern kein einheitliches Bild bei der multikriteriellen Bewertung zu erwarten ist, sich keine verwendungsunabhängig eindeutig zu präferierende Variante abzeichnet. Dies kann auch als Hinweis darauf interpretiert werden, bei zukünftigen Forschungs- und Innovationsstrategien auf nationaler Ebene mehrere technologische Optionen im
Blick zu behalten und nicht zu früh Entwicklungsaktivitäten zu fokussieren.

Diese Schlussfolgerung wird noch deutlicher, wenn man mit dem gleichen Instrumentarium einmal stationäre Anwendungen von Energiespeichern in den Blick nimmt. So konnten im Systemvergleich bei Techniken für die Sicherstellung einer unterbrechungsfreien Stromversorgung v. a. Lithium-Eisen-Phosphat- und Lithium-Mangan-Akkumulatoren überzeugen, während für einen Lastenausgleich innerhalb von Stromnetzen gegenwärtig Natrium-Schwe-

Abb. 3: Vergleich der Speichertechnologien*

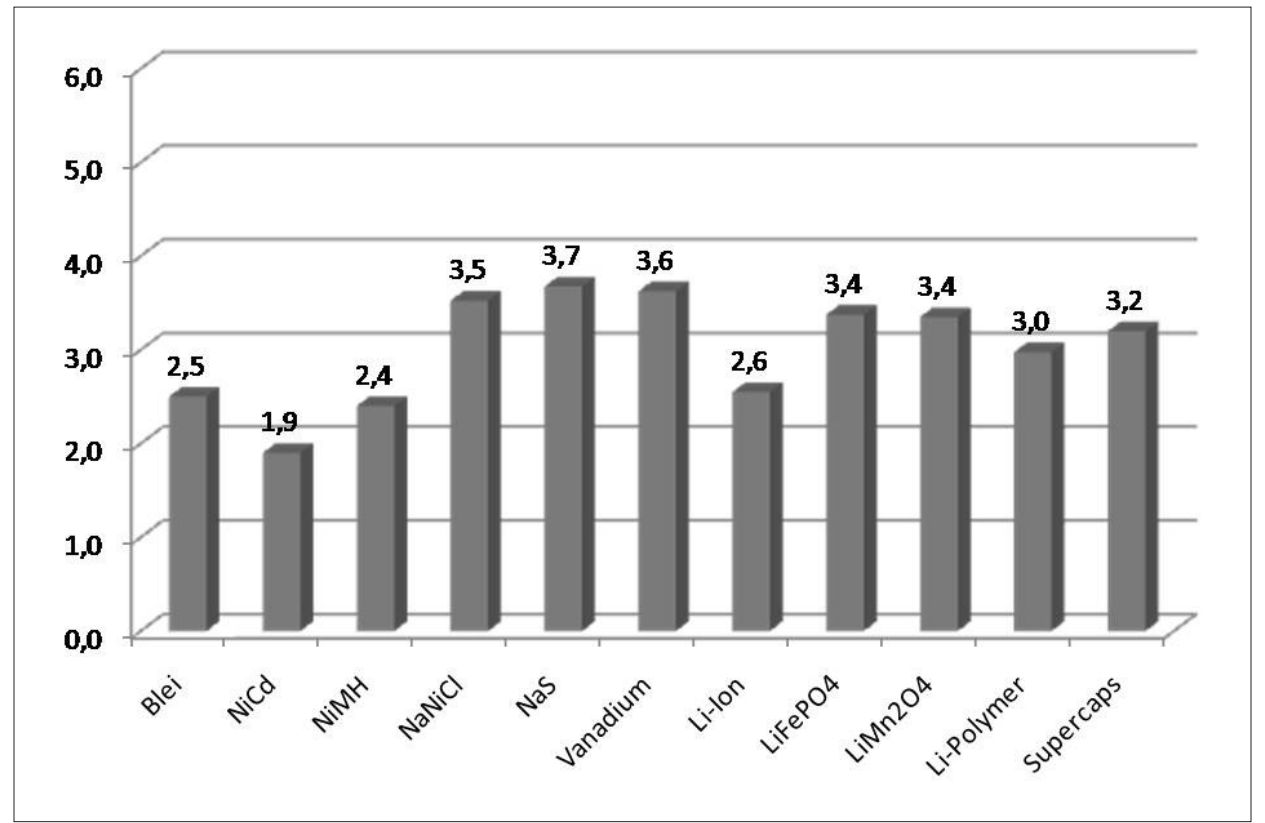

* = Die Y-Achse ist eine dimensionslosePunkteskala, vorteilhafte Energiespeichertechnologien weisen hohe Punkzahlen auf.

Quelle: Eigene Darstellung, auf der Basis von Frankenberg 2010 
fel-, Vanadium-Redox-Flow- und NatriumNickelchlorid-Akkumulatoren besonders vorteilhaft erscheinen.

Solche Überlegungen können natürlich nur eine Momentaufnahme darstellen. Welche Speichertechnologie sich in der Zukunft in den vorgestellten Anwendungsgebieten tatsächlich als geeignet erweisen wird, ist wesentlich abhängig von dem erreichbaren Technologiefortschritt: $\mathrm{Zu}$ arbeiten ist hier an einer höheren Leistungsfähigkeit (bei geringem Gewicht), an einer höherer Lebensdauer (kalendarisch, Be-/Entladezyklen, Temperatur) und nicht zuletzt an geringeren Kosten. Aktuell wird auch die Kombination einzelner Speichertechnologien intensiv diskutiert und entwickelt, was aus Sicht der Untersuchungsergebnisse nur unterstützt werden kann.

Weiter muss darauf hingewiesen werden, dass bei den oben vorgestellten multikriteriellen Bewertungen Umweltaspekte wie Recyclingfähigkeit (Weil 2010), Ressourcenverfügbarkeit (Weil et al. 2009) oder ökologische Gesamtperformance bisher nicht eingeflossen sind. Ebenso muss geklärt werden, wo Nanomaterialien in den elektrochemischen Speichern am Ende ihres Lebenszyklus verbleiben. Hierfür wäre unter anderem für alle Speichertypen im Anwendungskontext eine Lebenszyklusanalyse zu erstellen, um die Umweltverträglichkeit bewerten zu können. Dies sollte - in Übereinstimmung mit den obigen Überlegungen - nicht nur für das klassische Entwurfsparadigma für Fahrzeuge erfolgen, sondern auch den Vergleich zwischen unterschiedlichen Fahrzeugkonzeptionen ermöglichen.

Viele Hoffnungen auf eine Verbesserung der technischen und ökonomischen Parameter von Energiespeichern ruhen auf der Nutzung von Erkenntnissen der Nanowissenschaften, dem Einsatz von Nanomaterialien oder Nanotechnologie. Sowohl für mobile oder stationäre Anwendungen sollen sie helfen, die bisherigen Limitationen der bekannten elektrochemischen Systeme zu überwinden. Das betrifft dabei sowohl die aktiven Elemente wie Anode und Kathode, als auch die passiven Elemente wie Membranen (z. B. Separator).

Auch daraus können neue Fragestellungen für Technikfolgenabschätzung und Systemanalyse abgeleitet werden. Die eingesetzten Nano- materialien oder auf Nanotechnologie basierten Produkte haben zunächst v. a. beabsichtigte Wirkungen, etwa die Verbesserung der technischen Performance oder eine Reduktion der Systemkosten. Mit ihnen können aber auch neue unbeabsichtigte Folgen verbunden sein. So wird beispielsweise diskutiert, ob und bei welchen Materialen durch den Übergang in die Nanoskaligkeit neue Eigenschaften auftreten, die möglicherweise mit Umwelt- und Gesundheitsrisiken verbunden sind. Auch ist bisher weitgehend unbeantwortet, in welchem Verhältnis die Mehraufwendungen in der Herstellung solcher Materialien und Systeme (und ggf. auch bei ihrer Entsorgung) zur erwarteten technischen Leistungsverbesserung stehen. Solche Fragen werden - gemessen am Fördervolumen - in der Regel deutlich weniger intensiv untersucht und Ergebnisse diesbezüglicher Untersuchungen nur sehr eingeschränkt während der Entwicklungsphase berücksichtigt (Van den Bossche et al. 2006; Weil 2009). Dabei ist für eine rechtzeitige Diskussion dieser Probleme zu plädieren, könnten sie sich doch durchaus als Umsetzungshemmnisse erweisen.

\section{Elektrofahrzeuge im System der Energieversorgung}

Angesichts der gegenwärtig vorherrschenden und sehr wahrscheinlich noch für längere Zeit bestehenden bleibenden Struktur der Elektrizitätsversorgung kann davon ausgegangen werden, dass bei einem starken Wachstum der Elektromobilität Verkehrsnetze (mit Elektromobilität) und Energienetze zukünftig sehr stark interagieren werden. Welche neuen Anforderungen sich daraus für elektrochemische Speicher ergeben ist derzeit unklar.

Ein Thema, das dabei schon eine gewisse forschungspolitische und öffentliche Aufmerksamkeit erlangt hat, sind neue Interaktionen zwischen Elektrizitätsversorgern und Verbrauchern, die sich im Zuge einer stärkeren Marktdurchdringung von Elektromobilität herausbilden können. Dominik Möst und Kollegen diskutieren in ihrem Beitrag in diesem Heft ausführlich energiewirtschaftliche Fragen und Konsequenzen, die im Zusammenhang mit dieser Entwick- 
lung stehen (Möst et al. 2010). Sie nehmen dabei auch kurz Bezug auf das gegenwärtig intensiv diskutierte Konzept der (temporären) Nutzung von in Fahrzeugen integrierten Speichern als Betriebsmittel der Elektrizitätsversorgung, etwa zur Netzstabilisierung oder zum Ausgleich von Diskrepanzen zwischen Energienachfrage und Energieangebot. Dies ist auf den ersten Blick faszinierend, ließen sich damit doch zugleich Probleme der Netzintegration größerer Mengen fluktuierender regenerativer Energiequellen mindern und möglicherweise die ökonomischen Herausforderungen bei der Markteinführung von Elektrofahrzeugen reduzieren.

Aus Sicht der Technikfolgenabschätzung muss festgestellt werden, dass bei diesen Betrachtungen v. a. soziale und institutionelle Faktoren bisher nicht ausreichend untersucht und verstanden worden sind. So kann zum Beispiel nicht ohne weiteres davon ausgegangen werden, dass Fahrzeugeigentümer überhaupt dazu bereit sind, ihr System einem weitgehend anonymen Dritten zur Nutzung zu überlassen. Unter welchen Umständen sie es wären, und ob v. a. die in diesem Zusammenhang oft angeführten finanzielle Anreize überhaupt darstellbar sind, die dies für die Eigentümer attraktiv machen, ist offen. Schließlich ist eine Traktionsbatterie auf absehbare Zeit eine sehr teure Komponente im Fahrzeug, die durch Nutzung altert. Lässt sich dieser Wertverlust durch die Nutzung infolge einer zeitweiligen Netzeinbindung zugunsten des Elektrizitätsversorgers für den Kunden quantifizieren und ausreichend kompensieren? Wäre es möglicherweise eine auch aufgrund dieser Herausforderungen günstigere Strategie, Traktionsbatterien nicht mit fest mit dem Fahrzeug zu verbinden und in das Eigentum des Fahrzeugbesitzers übergehen zu lassen, sondern sie als wechselbare Komponente, die vom Elektrizitätsversorger vermietet wird, auszulegen? Es wäre zu diskutieren, inwiefern sich letztere Strategie nicht auch als potenzieller Lösungsansatz für „Nachtanken unterwegs“ einfacher umsetzen ließe als Ansätze, die auf das Schnellladen von Fahrzeugen setzen.

Ein zweites ungeklärtes Problem betrifft die Steuerung der Restkapazität des Speichersystems, das in die stationäre Elektrizitätsver- sorgung eingebunden wird. Kulturell sind Personenfahrzeuge auch Mobilitätsoptionen auf Vorrat. Die Möglichkeit, jederzeit und spontan mobil sein zu können, ist ein wichtiger Kaufanreiz für Individualverkehrsmittel. Inwieweit wird diese Option durch den (zeitweisen) Zugriff des Energieversorgers auf die Batterie beeinträchtigt - und sei es auch nur subjektiv - und wie kann dem durch intelligente Regelkonzepte zumindest teilweise begegnet werden? Zudem wäre zu beantworten, wer bei Nichteinhalten von entsprechenden Abreden zur Nutzung einer Traktionsbatterie oder gar im Schadensfall haftet.

\section{Nutzerperspektiven und -erwartungen}

Den Erwartungen und den Verhaltensweisen der zukünftigen Nutzer kommt für eine breite Durchsetzung der Elektromobilität eine entscheidende Rolle zu. Es liegt inzwischen eine ganze Zahl von aktuellen Studien vor, die sich einzelner damit verbundener Fragestellungen annehmen. Eine vergleichende Durchsicht dieser Arbeiten zeigt ein uneinheitliches und teilweise widersprüchlich anmutendes Bild. Beispielsweise nähert sich bei der Untersuchung von Zahlungsbereitschaften und Voraussetzungen für Kaufentscheidungen eine Gruppe von Studien aus dominant ökonomischer Perspektive und evaluiert v. a. das Kosten-Nutzen-Verhältnis. Der potenzielle Markterfolg von Elektrofahrzeugen wird hier eher kritisch gesehen, Hemmnisse v. a. in den höheren Anschaffungskosten und der geringen Reichweite gefunden. Manche Autoren fordern daher auch staatliche Subventionen für die Unterstützung der Marktdiffusion von Elektrofahrzeugen (z. B. ARIS 2010; Arnold et al. 2010), zumindest in der Frühphase. Andere Studien (z. B. RBSC 2010; Yetano Roche et 2009) geben an, in der Bevölkerung eine große Bereitschaft, finanzielle Einbußen zu Gunsten einer saubereren und zukunftsträchtigen Mobilität in Kauf zu nehmen, gezeigt zu haben.

Diese Widersprüchlichkeit, die hier nur als Beispiel für weitere uneinheitliche Befunde dienen soll, ist sicher in Teilen methodischen Schwächen und mutigen Interpretationen geschuldet. Die bisher vorliegenden Aussagen sind 
oft aus repräsentativen $\mathrm{CATI}^{2}$-Befragungen gewonnen, deren Design kaum dazu geeignet sind, kompliziertere Entscheidungskalküle zu eruieren. Es wäre Aufgabe der sozialwissenschaftlichen Technikforschung und der TA, in größerer Tiefe Fragen nachzugehen, was genau (und nicht nur im Bezug auf Kosten und Verbrauch) die Bürger an einem Auto schätzen, welche Eigenschaften (auch im Bezug auf Komfort, Design, etc. und nicht nur im Bezug auf Reichweite) sie sich von einem neuen Auto wünschen, für welche von ihnen als wichtig bewertete Eigenschaften sie welche Abzüge bei anderen Features in Kauf nehmen würden, welche Werte sie mit welcher Art von Mobilität verbinden usw. und wie sich Elektromobile hier im Vergleich zu konventionellen Fahrzeugen positionieren. Diese - und andere in diesem Kontext wichtige - Fragen sind sicher nicht immer neu, und von Instituten der Marktforschung auch schon ausführlich untersucht. Insgesamt muss das für die öffentliche Forschung zugängliche Wissen zu diesem Themenfeld aber immer noch als vergleichsweise dürftig bezeichnet werden. Das wird zur Herausforderung insbesondere dann, wenn daraus Resultate für die Beratung politischer Entscheidungsprozesse gewonnen werden sollen.

\section{Ausblick}

Realisierungsbedingungen und Folgen von Elektromobilität sind ein interessantes und - angesichts der Rolle des Individualverkehrs für die Gesellschaft insgesamt und der damit verbundenen Folgen - wichtiges Thema für die Technikfolgenabschätzung. Erste Stimmen sprechen bereits von einem unangemessen Hype des Themas, der bald einer Ernüchterung weichen wird. Zugleich sind aber viele mit der Einführung einer energiestrukturell neuartigen Mobilitätsoption verbundene Fragen, die ebendiese Einschätzung stützen oder widerlegen könnten, immer noch ungeklärt. Manche davon begleiten die Forschung schon seit geraumer Zeit, die aktuelle Debatte um Elektromobilität kann wissenschaftshistorisch als dritte oder vierte Welle seit den Ölpreiskrisen in den siebziger Jahren gelesen werden. Andere sind neu, da sich sowohl die technischen als auch die institutionellen Bedingungen über die letzten Jahrzehnte deutlich geändert haben.

Der vorliegende Beitrag hat versucht einige potenzielle Themenfelder für Technikfolgenabschätzung und Systemanalyse zur Elektromobilität aufzuzeigen. Aufgrund der Breite des Themengebietes konnten nicht alle interessanten Aspekte vertieft behandelt werden. Nicht weiter besprochen haben wir beispielsweise Akteurskonstellationen auf dem Gebiet der Elektromobilität (Kunden (,Verbraucher"), Automobilindustrie, Elektrizitätsversorgungsunternehmen sowie „die Politik“) mit ihren - teilweise sehr unterschiedlichen - Interessen und daraus entstehenden Umsetzungskonflikten. Ebenfalls nicht weiter ausgeführt wurden Überlegungen zu möglichen Ansatzpunkten für politisches Handeln zur Unterstützung der Umsetzung von Elektromobilität. Neben der klassischen Forschungsförderung betrifft dies v. a. mögliche Anreizmechanismen für zukünftige Käufer und/oder Nutzer von Elektrofahrzeugen sowie eine koordinierende oder moderierende Funktion bei Interessenkonflikten der Beteiligten.

Daneben sollten Technikfolgenabschätzung und Systemanalyse auch übergreifende Fragen wie die nach technischen Entwicklungspotenzialen bei konkurrierenden Speichersystemen oder nach eventuellen Ressourcenknappheiten bei Rohstoffen für Komponenten von Elektrofahrzeugen (Saskia Ziemann und KollegInnen diskutieren dies an anderer Stelle in diesem Heft ausführlicher (Ziemann et al. 2010)), die als Showstopper für Innovationsstrategien wirken könnten, weiter im Blick behalten.

\section{Anmerkungen}

1) Infolge der dynamischen Entwicklung auf diesem Gebiet sind Fachbücher zum Thema immer etwas „hinterher“. Für einen instruktiven Einblick in aktuelle Fahrzeugangebote und Designkonzepte empfehlen wir Websites wie http://www.grueneautos.com/elektroautos.php oder http://www. pluginamerica.org/vehicles sowie die einschlägige Automobilpresse.

2) CATI steht für „Computer Assisted Telephone Interview"; es handelt sich dabei um ein standardisiertes Erhebungsverfahren der empirischen Sozialforschung. 


\section{Literatur}

ARIS - ARIS Umfrageforschung Markt-, Mediaund Sozialforschungsgesellschaft $\mathrm{mbH}, 2010$ : Studie „Elektromobilität“. Umfrage im Auftrag von BITKOM - Bundesverband Informationswirtschaft, Telekommunikation und neue Medien e.V. April 2010

Arnold, H.; Kurtz, R.; Kuhnert, F.; Bauer, W., 2010: Elektromobilität - Herausforderungen für Industrie und öffentliche Hand. Fraunhofer IAO und PriceWaterhouseCoopers, Juni 2010

BMVBS - Bundesministerium für Verkehr, Bau und Stadtentwicklung, 2006: Nationales Innovationsprogramm Wasserstoff- und Brennstoffzellentechnologie. 8. Juni 2006; http://www.bmvbs.de/cae/servlet/contentblob/32980/publicationFile/1163/nationales-innovationsprogramm-wasserstoff-und-brennstoffzellen-technologie-nicht-barrierefre.pdf (download 1.12.10)

BMWi - Bundesministerium für Wirtschaft und Technologie, 2009: Auszug aus dem Bericht an den Haushaltausschuss Konjunkturpaket II, Ziffer 9 Fokus „Elektromobilität"; $\quad$ http://www.foerderinfo.bund. de/_media/elektromobilitaet_konjunkturpaket_ii.pdf (download 1.12.10)

Bundesregierung, 2009. Nationaler Entwicklungsplan Elektromobilität der Bundesregierung. August 2009; http://www.bmwi.de/Dateien/BMWi/PDF/nationalerentwicklungsplan-elektromobilitaet-der-bundesregie rung,property $=$ pdf, bereich $=$ bmwi, sprache $=d e, r w b=\operatorname{tr}$ ue.pdf (download 1.12.10)

Böse, O., 2009: Aging Behaviour of Ultra High Power Li-Ion Batteries. Continental AG. Advanced Battery Technology, Frankfurt 07/2009

Frankenberg, A., 2010: Vergleich und Bewertung von elektrochemischen Energiespeichern mit Schwerpunkt auf den Lithium-Akkumulatoren. KIT-Diplomarbeit

Möst, D.; Jochem, P.; Fichtner, W., 2010: Dezentralisierung der Energieversorgung. Herausforderungen an die Systemanalyse und -steuerung. In: Technikfolgenabschätzung - Theorie und Praxis 19/3 (2010), S. 22-29

Oertel, D., 2008: Energiespeicher - Stand und Perspektiven. Sachstandsbericht zum Monitoring „Nachhaltige Energieversorgung“. TAB-Arbeitsbericht Nr. 123. Berlin RBSC - Roland Berger Strategy Consultants, 2010: Powertrain 2020. Electric Vehicles - Voice of the Customer. München

Yetano Roche, M.; Mourato, S.; Fischedick, M. et al., 2010: Public Attitudes Towards and Demand for Hydrogen and Fuel Cell Vehicles: A Review of the Evidence and Methodological Implications. In: Energy Policy 38/10 (2010), S. 5301-5310
Van den Bossche, P.; Vergels, F.; Van Mierlo, J. et al., 2006: SUBAT: An Assessment of Sustainable Battery Technology. In: Journal of Power Sources, 162/2 (2006), S. 913-919

Weil, M., 2009: Dealing with Unknowns - How to Optimise Nano Material Production. Industrial Ecology, ISIE conference, Lissabon, 06/2009

Weil, M.; Ziemann, S.; Schebek, L., 2009: How to Assess the Availability of Resources for New Technologies? Case Study: Lithium, a Strategic Metal for Emerging Technologies. In: Revue de Metallurgie 12 (2009), S. 554-558

Weil, M., 2010: Future Availability of Lithium - Does Recycling Help? 3rd International Congress on Advanced Battery Technology. Wiesbaden, 06/2010

Ziemann, S.; Weil, M.; Schebek, L., 2010: Energietechnologien der Zukunft - die Problematik der Rohstoffverfügbarkeit am Beispiel von Lithium. In: Technikfolgenabschätzung - Theorie und Praxis 19/3 (2010), S. 46-55

\section{Kontakt}

Dipl.-Phys. Torsten Fleischer

Karlsruher Institut für Technologie (KIT)

Institut für Technikfolgenabschätzung und

Systemanalyse (ITAS)

Campus Nord

Hermann-von-Helmholtz-Platz 1

76344 Eggenstein-Leopoldshafen

Tel.: +49 (0) 7247 / 82 - 4571

E-Mail: torsten.fleischer@kit.edu 